\title{
Treatment with PPARd agonist alleviates non-alcoholic fatty liver disease by modulating glucose and fatty acid metabolic enzymes in a rat model
}

\author{
XIULI LI ${ }^{1}$, JIN LI $^{2,3}$, XIAOLAN LU ${ }^{2}$, HUIHUI MA ${ }^{2}$, HAITAO SHI ${ }^{2}$, \\ HONG LI $^{2}$, DANHONG XIE ${ }^{2}$, LEI DONG ${ }^{2}$ and CHUNLIAN LIANG ${ }^{1}$ \\ Departments of ${ }^{1}$ Geriatrics and ${ }^{2}$ Gastroenterology, The Second Affiliated Hospital, Medical School \\ of Xi'an Jiaotong University; ${ }^{3}$ Department of Gastroenterology, Xi'an Central Hospital, \\ Medical School of Xi'an Jiaotong University, Xi'an, Shaanxi 710004, P.R. China
}

Received October 25, 2014; Accepted June 11, 2015

DOI: $10.3892 / \mathrm{ijmm} .2015 .2270$

\begin{abstract}
Non-alcoholic fatty liver disease (NAFLD) is an increasingly common condition which is associated with certain features of metabolic syndrome and insulin resistance. Peroxisome proliferator-activated receptor (PPAR) $\delta$ is an important regulator of energy metabolism and insulin resistance in diabetes. However, the function of PPAR $\delta$ in NAFLD has not yet been fully elucidated. In the present study, in order to explore the function of PPAR $\delta$ in NAFLD, we created a rat model of NALFD induced by a high-fat diet (HFD) and treated the rats with GW501516, a PPAR $\delta$ agonist. We found that the lipid levels decreased, and hepatocellular ballooning and inflammatory cell infiltration were also significantly decreased following treatment of the rats with GW501516 compared to the untreated rats. Treatment with GW501516 also significantly decreased the homeostasis model assessment of insulin resistance (HOMA-IR) index, as well as the low-density lipoprotein (LDL) levels. In addition, treatment with GW501516 increased the levels of insulin-like growth factor-1 (IGF-1) and high-density lipoprotein (HDL) compared to the HFD group. Furthermore, the elevated levels of alanine aminotransferase (ALT), aspartate aminotransferase (AST), gamma-glutamyl transpeptidase (GGT) and alkaline phosphatase (ALP) in the HFD group were all restored to the normal control levels following treatment with GW501516. RT-qPCR and immunohistochemical staining revealed that the expression levels of sterol regulatory element binding protein-1c (SREBP-1c) and glucose transporter 2 (GLUT-2)
\end{abstract}

Correspondence to: Dr Xiaolan Lu, Department of Gastroenterology, The Second Affiliated Hospital, Medical School of Xi'an Jiaotong University, No. 157 West 5th Road, Xi'an, Shaanxi 710004, P.R. China E-mail: xiaolanlus@163.com

Key words: non-alcoholic fatty liver disease, peroxisome proliferatoractivated receptor $\delta$, GW501516, insulin resistance enzymes related to lipid metabolism were both restored to normal control levels following treatment with GW501516. Also, the levels of enzymes related to lipid metabolism were increased following treatment with GW501516. In conclusion, our findings demonstrate that treatment with GW501516 alleviates NAFLD by modulating glucose and fatty acid metabolism.

\section{Introduction}

Non-alcoholic fatty liver disease (NAFLD) is characterized by fat accumulation in the liver that is not due to alcohol abuse. Epidemiological data indicate that NAFLD is not simpl a liver disease, but rather a concentrated reflection of obesity, hyperlipidemia and insulin resistance and other metabolic disorders in the liver $(1,2)$. Although low-grade NAFLD is considered a benign disease, without appropriate treatment, it can cause more severe liver injury, and may develop into non-alcoholic steatohepatitis, fibrosis, cirrhosis, and in some cases, hepatocellular carcinoma $(3,4)$. Previous studies have revealed that NAFLD is more prevalent in obese individuals and patients with type 2 diabetes $(5,6)$, suggesting an association between metabolic syndrome and insulin resistance (1,4,7-9). However, the pathogenesis of NAFLD is not yet fully understood, and an effective treatment for NAFLD is yet to be developed.

Peroxisome proliferator-activated receptors (PPARs) are members of the steroid hormone nuclear receptor family (10). The PPAR family encompasses PPAR $\alpha, \operatorname{PPAR} \gamma$ and PPAR $\beta / \delta$, which are all ligand-activated transcription factors that mediate the hormonal control of gene expression. PPARs serve as important modulators in glucose and fatty acid metabolic pathways, as well as in adipocyte proliferation, differentiation and apoptosis (11-14). Agonists of PPARs have been shown to be effective in the treatment of metabolic syndrome, such as type 2 diabetes mellitus and NAFLD $(15,16)$. Thiazolidinediones (TZDs), a group of agonists of PPAR $\gamma$, have been widely studied and used in the treatment of type 2 diabetes since 1997 (15). Of these, pioglitazone, a selective agonist to the nuclear receptor PPAR $\gamma$ and to a lesser extent PPAR $\alpha$, has been shown to improve insulin resistance $(17,18)$. PPARs are considered to have medical significance (19). Another PPAR $\gamma$ agonist, rosiglitazone, has 
been shown to ameliorate non-alcoholic steatohepatitis (20). PPAR $\gamma$ has also been shown to regulate genes involved in insulin/insulin-like growth factor signaling, lipid metabolism and adipocyte-specific development (21).

PPARS is ubiquitously expressed in skeletal muscle cells, as well as in liver cells $(22,24)$. It has been documented that GW501516, a potent agonist to PPAR $\delta$, modulates glycometabolism, fatty acid metabolism and alleviates insulin resistance (22-24). GW501516 increases fatty acid oxidation by increasing the levels of hepatic mRNAs associated with fatty acid $\beta$-oxidation, such as acyl-CoA oxidase (ACOX), carnitine palmitoyltransferase-1 (CPT-1) and liver fatty acid binding protein (L-FABP) $(23,25)$. The increase in fatty droplets within hepatocytes and the development of steatohepatitis in mice fed a methionine- and choline-deficient diet has been shown to be inhibited by GW501516 (25). However, the effects of GW501516 on NAFLD remain controversial, and the underlying mechanisms remain to be elucidated.

In the present study, we explored the effects of GW501516 in a rat model of NAFLD. It was found that GW501516 effectively alleviated NAFLD. Glucose and fatty acid metabolism, as well as the related enzymes, were also found to be regulated by GW501516.

\section{Materials and methods}

Animals and treatment. Forty-six 8-week-old male Sprague-Dawley (SD) rats obtained from the Laboratory Animal Center of Xi'an Jiaotong University (Xi'an, China) were used in the present study. All the rats were housed under specific pathogen-free conditions with free access to water. All the experiments were carried out in adherence to a protocol approved by the Institutional Animal Care and Use Committee of Xi'an Jiaotong University. Of the 46 rats, some were fed a standard chow diet (Laboratory Animal Center of Xi'an Jiaotong University, Xi'an, China) and were assigned to the normal control group $(n=10)$, while the others were used to establish a model of NAFLD. For the induction of NAFLD, the rats were fed a high-fat diet (HFD; 10\% lard, $2 \%$ cholesterol, $5 \%$ saccharose, $0.5 \%$ hog bile extract and $82.5 \%$ standard chow diet) for 12 weeks. The 36 rats fed the HFD were assigned to the HFD control group $(n=12)$, the GW501516 treatment group ( $\mathrm{n}=12)$, or the pioglitazone treatment group $(\mathrm{n}=12)$. Therapeutic intervention with GW501516 (Santa Cruz Biotechnology, Santa Cruz, CA, USA) or pioglitazone (Santa Cruz Biotechnology) was initiated after 8 weeks on the HFD. GW501516 (10 mg/kg body weight/day, in water) and pioglitazone $(10 \mathrm{mg} / \mathrm{kg}$ body weight $/ \mathrm{day}$, in water) were administered orally once a day to the rats in the respective groups from the 8th week until the 12th week. The rats in the HFD control group received the same amount of normal saline. Blood samples were collected from the tail vein and stored at $-70^{\circ} \mathrm{C}$ until biochemical analysis. At the end of each experiment, the rats were sacrificed by an intravenous administration of pentobarbital (overdose). The body weight, liver wet weight and body length of the rats were then measured. The hepatosomatic index was calculated as follows: hepatosomatic index $=$ liver wet weight $(\mathrm{g}) /$ body weight $(\mathrm{g})$. The body mass index was calculated as follows: body mass index $=$ body weight $(\mathrm{kg}) /$ body length $(\mathrm{m})^{2}$. The livers were cut into a number of sections and stored at $-80^{\circ} \mathrm{C}$ until tissue examination.

Biochemical assays. Serum fasting blood glucose (FBG), triglyceride (TG), total cholesterol (TC), low-density lipoprotein (LDL), high-density lipoprotein (HDL), alanine aminotransferase (ALT), aspartate aminotransferase (AST), gamma-glutamyl transpeptidase (GGT) and alkaline phosphatase (ALP) levels were measured using an automatic biochemical analyzer. The serum insulin levels were measured using an enzyme-linked immunosorbent assay (ELISA) kit (EIA2048; DRG International, Inc., Springfield, NJ, USA) according to the manufacturer's instructions. Insulin resistance was calculated using the homeostasis model assessment of insulin ressistance (HOMA-IR) as follows: fasting insulin (mIU/l) $\mathrm{x}$ fasting blood glucose $(\mathrm{mmol} / \mathrm{l}) / 22.5$, as previously described (26). The serum insulin-like growth factor-1 (IGF-1) levels were measured using an ELISA kit (IB39431) purchased from IBL (Minneapolis, MN, USA), according to the manufacturer's instructions.

Histological analyses. Histological analyses were performed according to a the methods described in a previous study (27). Briefly, the liver tissues were fixed in $10 \%$ buffered formaldehyde and then embedded in paraffin. The paraffin-embedded tissues were cut into 5- $\mu \mathrm{m}$-thick sections and were then stained with hematoxylin and eosin (H\&E). To visualize the accumulation of fat droplets, sections of frozen liver tissue were stained with Oil Red O, and hematoxylin was introduced to labelmark the cell nuclei.

Immunohistochemical staining. The 5- $\mu \mathrm{m}$-thick paraffinembedded sections of liver tissue were deparaffinized by changing the xylene twice, and this procedure was followed by rehydration in an ethanol gradient. Following antigen retrieval and non-specific binding blocking, the slides were incubated with primary antibodies diluted at 1:800 for $60 \mathrm{~min}$ at $37^{\circ} \mathrm{C}$. After washing 3 times, the slides were then incubated with HRP-conjugated rabbit anti-rat IgG (Cat. no. ab6734; Abcam, Cambridge, UK) diluted at 1:500 for $60 \mathrm{~min}$ at $37^{\circ} \mathrm{C}$. Color development was performed using a DAB Horseradish Peroxidase Color Development kit (Beyotime, Nantong, China). Hematoxylin was used for nuclear compartment visualization. Rabbit anti-rat sterol regulatory element binding protein-1c (SREBP-1c) and glucose transporter type 2 (GLUT-2) antibodies were purchased from Santa Cruz Biotechnology. The results were quantified using a color density assay using iVision software (version 4.0.9; BioVision Technologies, Exton, PA, USA).

$R N A$ extraction and quantitative-polymerase chain reaction $(R T-q P C R)$. Total RNA from the rat livers was extracted using TRIzol reagent (Invitrogen, Carlsbad, CA, USA) according to the manufacturer's instructions. cDNA was synthesized from $5 \mu \mathrm{g}$ of the total RNA using a PrimeScript ${ }^{\circledR}$ RT reagent kit (Takara, Dalian, China) with oligo(dT) primers as per the manufacturer's instructions. The quantitative PCR (qPCR) reactions were performed on a Bio-Rad iQ5 real-time thermal cycler using a SYBR ${ }^{\circledR}$ Premix Ex Taq ${ }^{\mathrm{TM}}$ II kit (Takara) in a $25-\mu 1$ reaction system. All reactions were performed in triplicate, and the results are represented as relative mRNA 

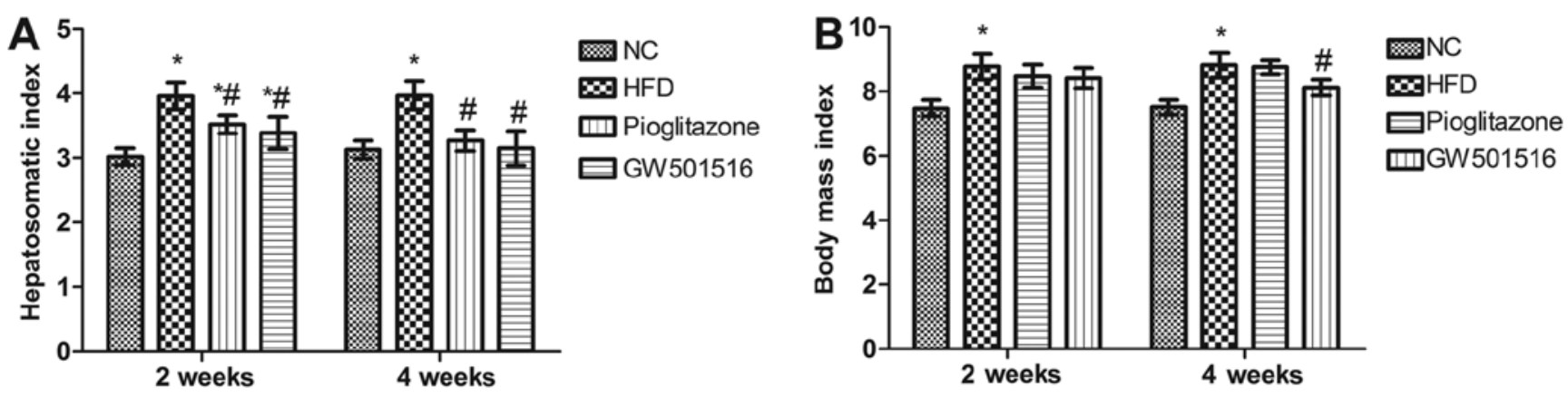

Figure 1. (A) Hepatosomatic index and (B) body mass index were calculated according to the body weight, liver wet weight and body length. All data are

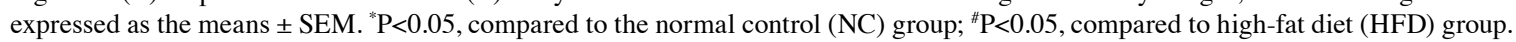

expression data, which were calculated using the $2^{-\Delta \Delta \mathrm{CT}}$ method, as previously described (28). The sequences of the primers used were as follows: SREBP-1c forward, 5'-CGC TAC CGT TCC TCT ATC AAT GAC-3' and reverse, 5'-AGT TTC TGG TTG CTG TGC TGT AAG-3'; GLUT-2 forward, 5'-TCC GCT TGC TCC TCC TCC TAC-3' and reverse, 5'-ACA CCGA TGT CAT ATC CGA ACT GG-3'; diacylglycerol acyltransferase (DGAT) forward, 5'-TCC GCC TCT GGG CAT TC-3' and reverse, 5'-GAA TCG GCC CAC AAT CCA-3'; CPT-1 forward, 5'-TAT GTG AGG ATG CTG CTT CC-3' and reverse, 5'-CTC GGA GAG CTA AGC TTG TC-3'; ACOX1 forward, 5'-GTT GAT CAC GCA CAT CTT GGA-3' and reverse, 5'-TCG TTC AGA ATC AAG TTC TCA ATT TC-3'; ACOX3 forward, 5'-TGG AGA AGG AAC GAG AAC TGA AC-3' and reverse, 5'-ACA TGT GGA GGA CAC ATT TGT TG-3'; and $\beta$-actin forward, 5'-CAA CTT GAT GTA TGA AGG CTT TGG T-3' and reverse, 5'-ACT TTT ATT GGT CTC AAG TCA GTG TAC AG-3'. All the primers were synthesized by BGI Tech (Shenzhen, China).

Statistical analysis. Data are expressed as the means \pm SEM from a minimum of 3 experiments. Differences between groups were analyzed using the Student's t-test. A value of $\mathrm{P}<0.05$ was considered to indicate a statistically significant difference.

\section{Results}

Hepatosomatic index and alterations in body mass index. Pioglitazone, a prescription drug of the class TZD, which is used in the treatment of diabetes, was introduced in the present study as a positive control. After 12 weeks of being fed the HFD, the hepatosomatic index and body mass index of the rats were both significantly elevated $(\mathrm{P}<0.05)$ compared to the rats in the normal control group (Fig. 1). However, 2 weeks of treatment with GW501516 or pioglitazone significantly reduced the hepatosomatic index $(\mathrm{P}<0.05)$ compared to the HFD group, although the hepatosomatic index was still significantly higher than that of the normal control group $(\mathrm{P}<0.05$; Fig. 1A). After 4 weeks of treatment with GW501516 or pioglitazone, the hepatosomatic index was almost reduced to the level of the normal control group (Fig. 1A). Treatment with pioglitazone did not reduce the body mass index (Fig. 1B). However, a significant decrease in body mass index was observed in the rats that were treated with GW501516 for 4 weeks (Fig. 1B).
Alleviation of pathological changes in the liver following treatment with GW501516. After 4 weeks of treatment with normal saline, GW501516 or pioglitazone, the livers of the rats in all the groups were collected and analyzed using Oil Red O (Fig. 2A) and H\&E (Fig. 2B) staining. The histological examination of the livers of the rats from the normal control group exhibited a normal structure and architecture. No obvious lipid droplets or lobular inflammatory cell infiltration were observed (Fig. 2). However, typical vesicular steatosis, hepatocellular ballooning, and lipid droplets were observed in the HFD group (Fig. 2). After 4 weeks of treatment with GW501516 or pioglitazone, these pathological changes were significantly alleviated. In addition, it was clear that GW501516 had a more potent effect than pioglitazone, as lipid droplets, hepatocellular ballooning and inflammatory cell infiltration were scarcely observed in the group treated with GW501516 (Fig. 2).

Effect of GW501516 on glucose metabolism. In order to investigate whether treatment with GW501516 affects glucose metabolism, serum was collected from the rats at the end of each treatment for the determination of the levels of fasting blood glucose, fasting insulin and IGF-1. The HOMA-IR index was calculated based on the levels of fasting blood glucose and fasting insulin. As expected, a significant increase in the serum levels of fasting blood glucose and fasting insulin, as well as in the HOMA-IR index was observed in the rats in the HFD group compared to the rats of the normal control group. In addition, a significant decrease in the levels of IGF-1 was observed in the HFD group compared to the normal control group (Fig. 3). However, 4 weeks of treatment with pioglitazone significantly decreased the levels of fasting blood glucose and fasting insulin, and the HOMA-IR index, and increased the levels of IGF-1 compared to the HFD group (Fig. 3). Nonetheless, these levels and indexes still differed significantly from those of the normal control group (Fig. 3). However, 4 weeks of treatment with GW501516 almost restored these indexes to normal levels (Fig. 3). This suggested that glucose metabolism is regulated by GW501516.

Effect of GW501516 on lipid metabolism. To determine the effect of GW501516 on lipid metabolism, the levels of TG, TC, HDL and LDL in the serum were measured. In contrast to the normal control group, the levels of TG, TC and LDL were 

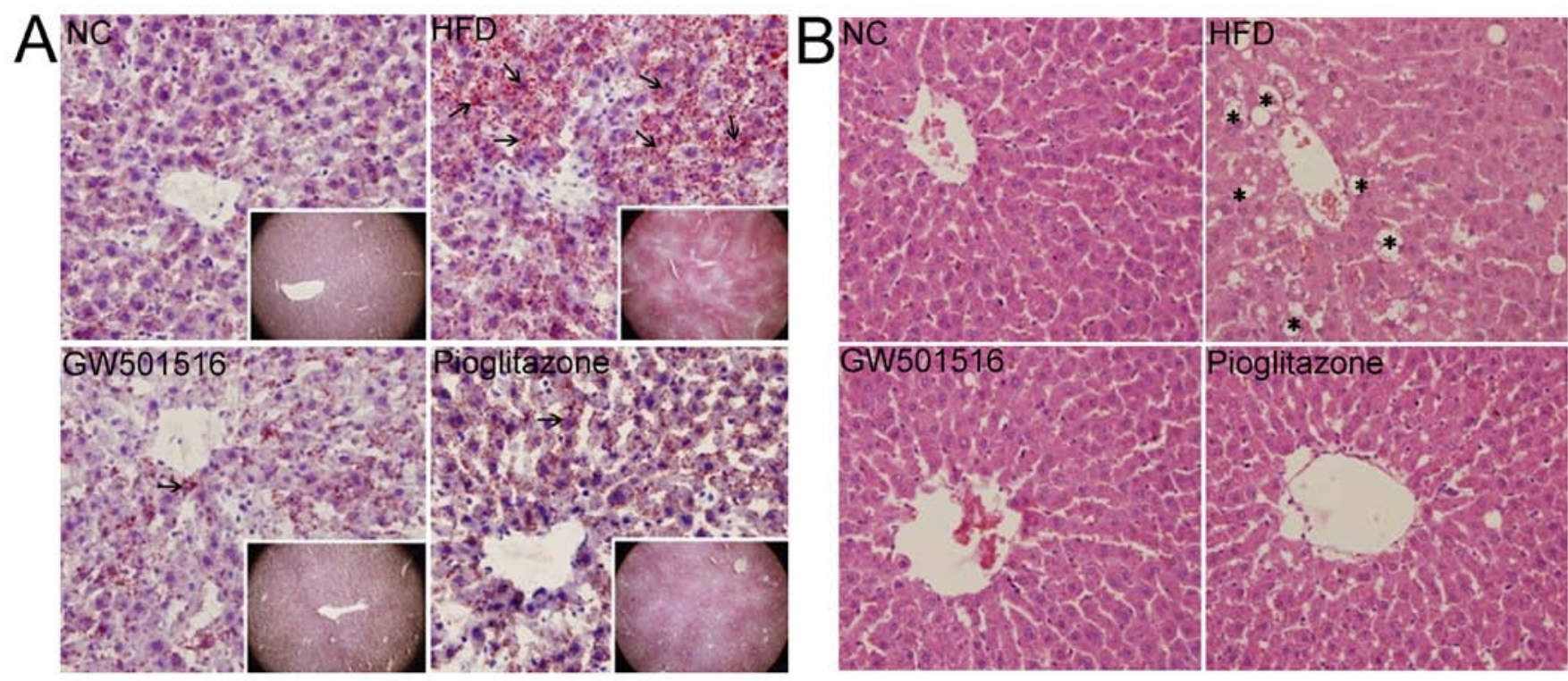

Figure 2. Histological changes in the rat livers after 4 weeks of treatment. (A) Oil Red O staining of lipid droplets counterstained with hematoxylin (blue) for the nuclei in cryosections of rat livers with different treatments. Arrows indicate lipid droplets. The insets show images of x10 magnification. (B) H\&E staining of paraffin-embedded sections of rat livers. Asterisks indicate hepatocellular ballooning. Original magnification, x400. NC, normal control; HFD, high-fat diet.
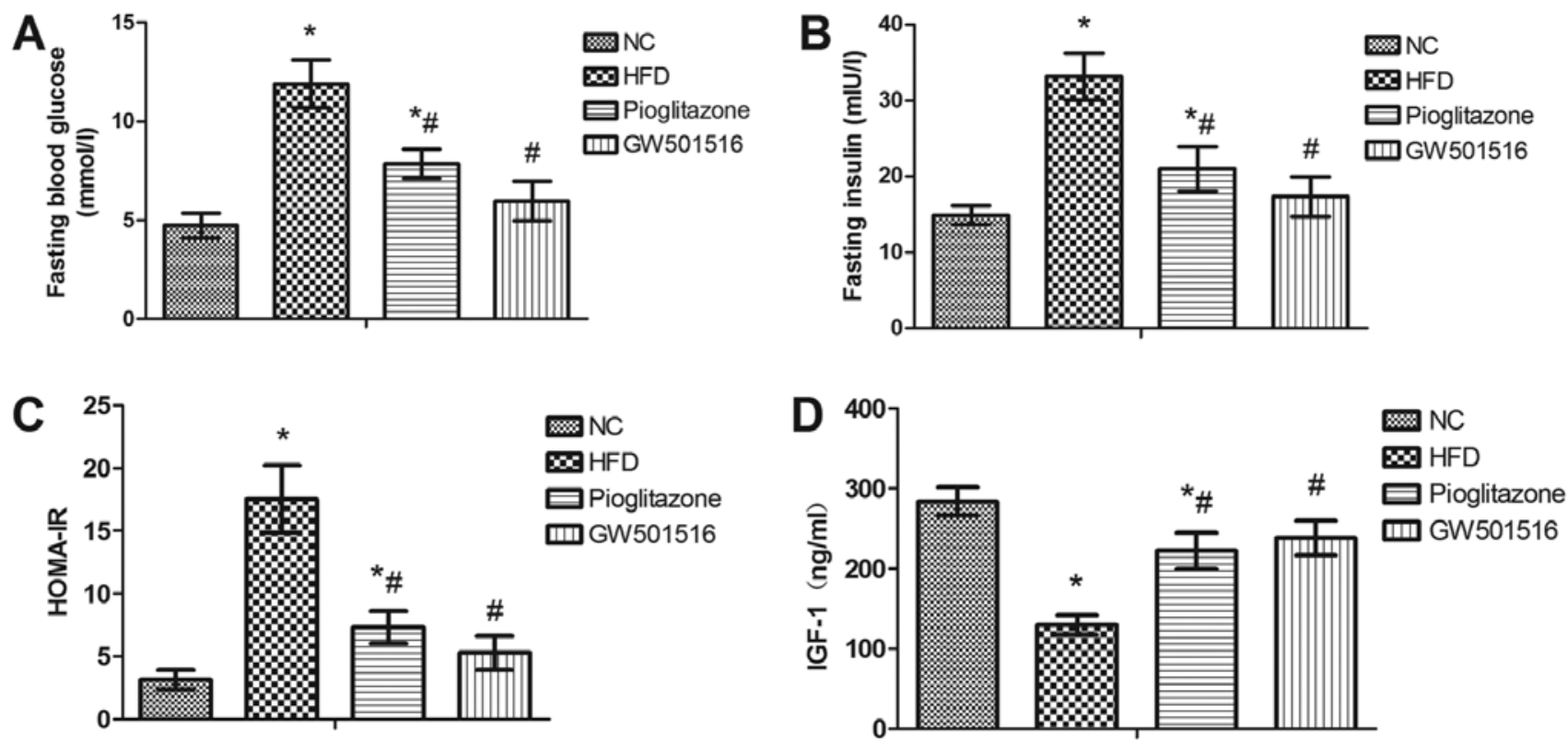

Figure 3. Changes in glucose metabolism indexes in rat serum after 4 weeks of treatment. (A) Fasting blood glucose and (B) fasting insulin levels were measured using an automatic biochemical analyzer. (C) The homeostasis model assessment of insulin resistance (HOMA-IR) index was calculated as follows: fasting insulin (mIU/l) x fasting blood-glucose (mmol/l)/22.5. Fasting blood glucose levels were measured using an automatic biochemical analyzer. Fasting insulin levels were determined using an ELISA kit. (D) Insulin-like growth factor-1 (IGF-1) levels were determined using an ELISA kit. All data are expressed as the means \pm SEM. ${ }^{*} \mathrm{P}<0.05$, compared to the normal control (NC) group; ${ }^{*} \mathrm{P}<0.05$, compared to the high-fat diet (HFD) group.

all significantly increased, and the levels of HDL were significantly decreased in the HFD group (Fig. 4). However, 4 weeks of treatment with pioglitazone significantly decreased the levels of TG, TC and LDL, and increased the levels of HDL in comparison to the HFD group (Fig. 4). Treatment with GW501516 almost restored the TG, TC, LDL and HDL levels to normal levels (Fig. 4). This suggested that lipid metabolism may be regulated by GW501516.
Effect of GW501516 on the levels of liver enzymes. To evaluate liver function, the serum levels of ALT, AST, GGT and ALP were determined. As shown in Fig. 5, the levels of ALT, AST, GGT and ALP were all significantly elevated in the HFD group compared to those of the normal control group, and these were all markedly reduced after 4 weeks of treatment with GW501516 compared to the HFD group (Fig. 5). These data indicate that GW501516 effectively alleviates NAFLD and that it is more 

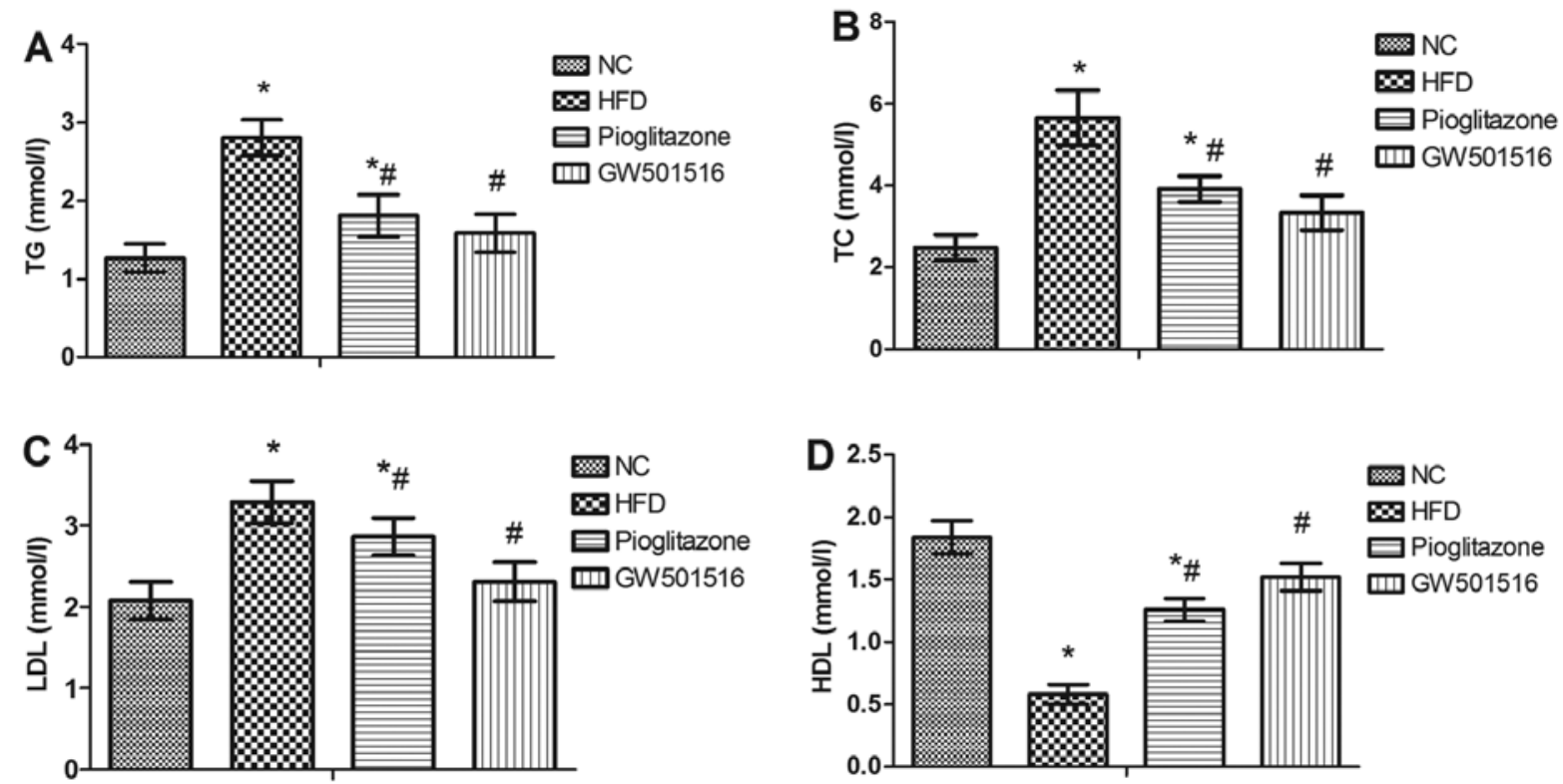

Figure 4. (A) Triglyceride (TG), (B) total cholesterol (TC), (C) low-density lipoprotein (LDL) and (D) high-density lipoprotein (HDL) levels were measured using an automatic biochemical analyzer. All data are expressed as teh means \pm SEM. ${ }^{*} \mathrm{P}<0.05$, compared to the normal control (NC) group; ${ }^{\sharp} \mathrm{P}<0.05$, compared to the high-fat diet (HFD) group.
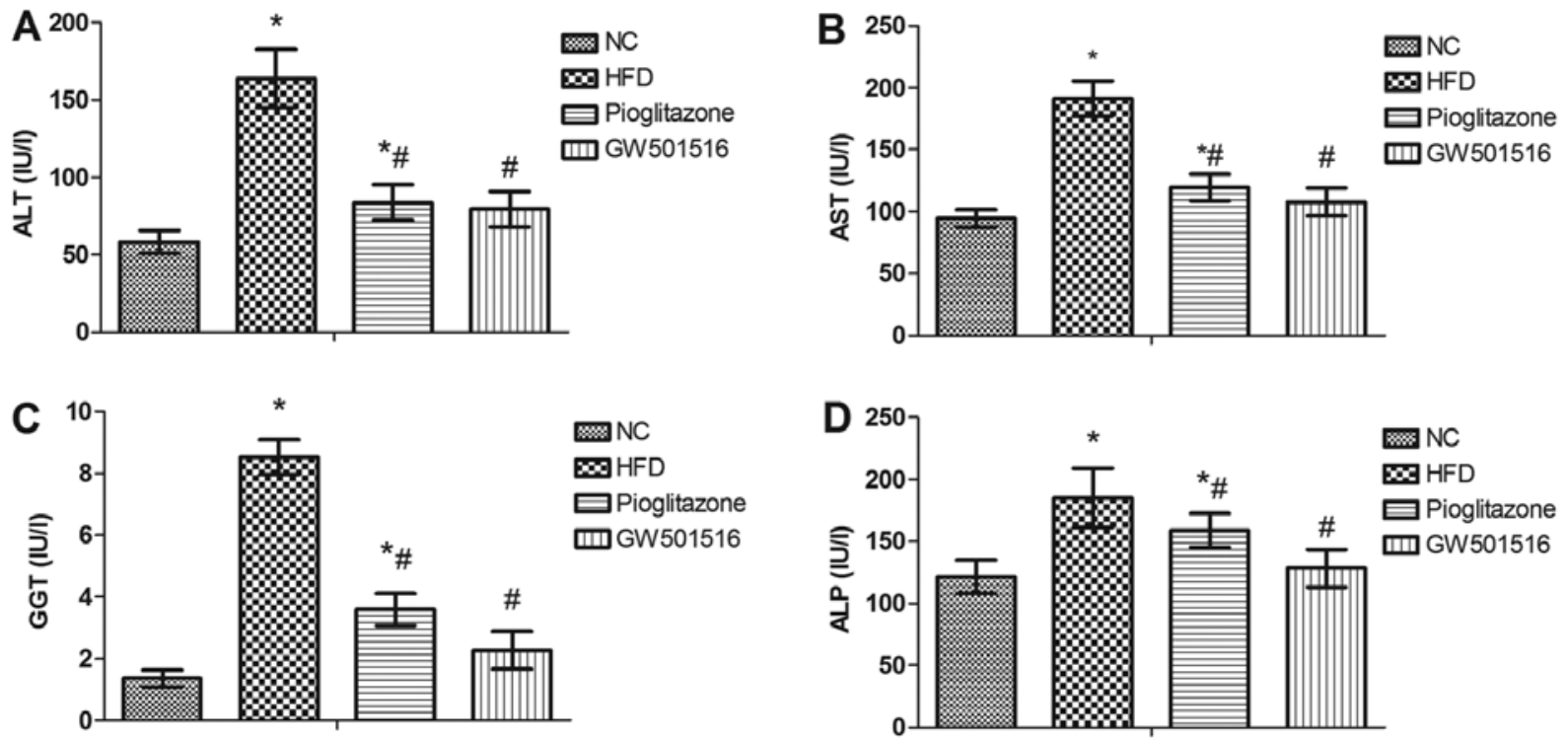

Figure 5. (A) Alanine aminotransferase (ALT), (B) aspartate aminotransferase (AST), (C) gamma-glutamyl transpeptidase (GGT) and (D) alkaline phosphatase (ALP) levels were measured using an automatic biochemical analyzer. All data are expressed as the means \pm SEM. "P $<0.05$, compared to the normal control (NC) group; ${ }^{\#} \mathrm{P}<0.05$, compared to the high-fat diet (HFD) group.

effective than pioglitazone, since treatment with pioglitazone did not reduce the ALT, AST, GGT or ALP levels to the same extent.

Expression of SREBP-1c and GLUT-2 is restored following treatment with GW501516. The above-mentioned findings indicate that treatment with GW501516 regulates glucose and lipid metabolism. To elucidate the underlying mechanisms responsible for the effects of GW501516 on glucose and lipid metabolism, the expression levels of SREBP-1c, a key lipogenic transcription factor involved in lipogenesis $(29,30)$, and GLUT-2 were determined. As expected, the mRNA levels of SREBP-1c were significantly upregulated and those of GLUT-2 were significantly downregulated in the livers of the rats in the HFD group compared to the normal control group (Fig. 6A and B). However, these levels were both restored to almost normal levels after 4weeks of treatment with GW501516 (Fig. 6A and B). A similar trend was observed in relation to the protein expression of SREBP-1c and GLUT-2, as was determined by immunohistochemical staining (Fig. 6C and D). These data suggest that GW501516 modulates glucose and lipid metabolism by regulating SREBP-1c and GLUT-2 expression. 

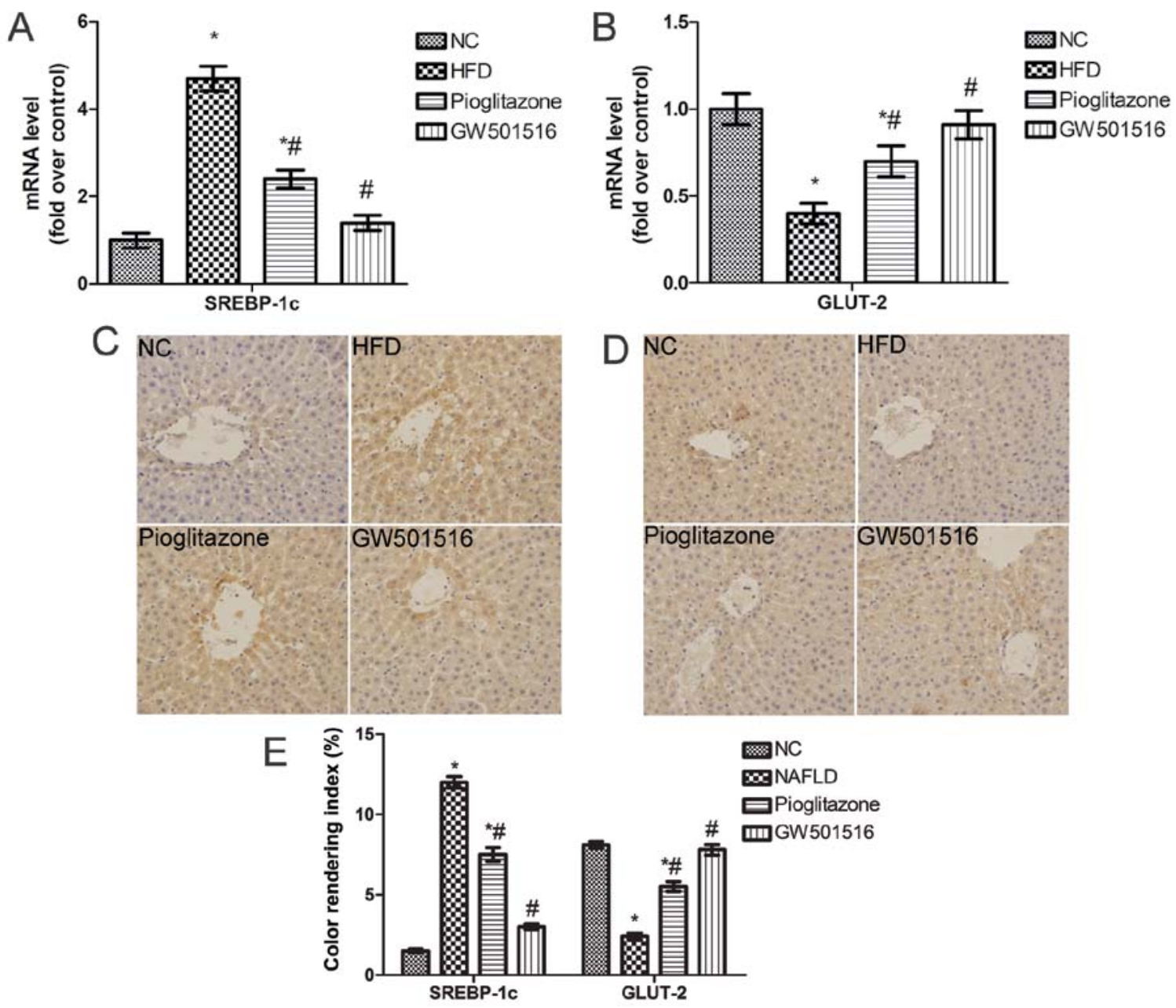

Figure 6. Effect of GW501516 on the expression of sterol regulatory element binding protein-1c (SREBP-1c) and glucose transporter type 2 (GLUT-2). The mRNA levels of (A) SREBP-1c and (B) GLUT-2 were determined by RT-qPCR. All data were normalized based on $\beta$-actin levels and are expressed as the means \pm SEM. Protein expression of (C) SREBP-1c and (D) GLUT-2 in paraffin-embedded rat liver tissues was analyzed by immunohistochemical staining (original magnification x400). (E) Immunohistochemical staining results of SREBP-1c and GLUT-2 were quantified using a color density assay and iVision software. Data are expressed as the means \pm SEM. ${ }^{*} \mathrm{P}<0.05$, compared to the normal control (NC) group; ${ }^{*} \mathrm{P}<0.05$, compared to the high-fat diet (HFD) group.

Expression of genes related to fatty acid synthesis is regulated by GW501516. Previous studies have demonstrated that the expression of genes related to lipid metabolism is modulated by PPARs (31-35), and it has been shown that the expression of SREBP-1c, a key lipogenic transcription factor, is regulated by GW501516 (36). As DGAT, CPT-1, ACOX1 and ACOX3 are important rate-limiting enzymes which are related to lipid metabolism, we determined the transcriptional levels of these genes in order to evaluate the effects of GW501516 on lipid metabolism. As shown in Fig. 7, the transcriptional levels of DGAT1, CPT-1 and ACOX1 were all significantly upregulated; however, those of ACOX3 were only slightly altered by the HFD. Four weeks of treatment with GW501516 did not greatly alter the transcription levels of DGAT1, in comparison to those of the HFD group. This suggests that triglyceride synthesis is not significantly affected by GW501516, as DGAT1 is the rate-limiting enzyme for the synthesis of TGs from diacylglycerols (37). However, the transcriptional levels of CPT-1, ACOX1 and ACOX3 were significantly upregulated following tretment with GW501516 compared to the levels of the HFD group (Fig. 7). These data suggest that the $\beta$-oxidation of fatty acids in the mitochondria and peroxisomes is improved by treat-

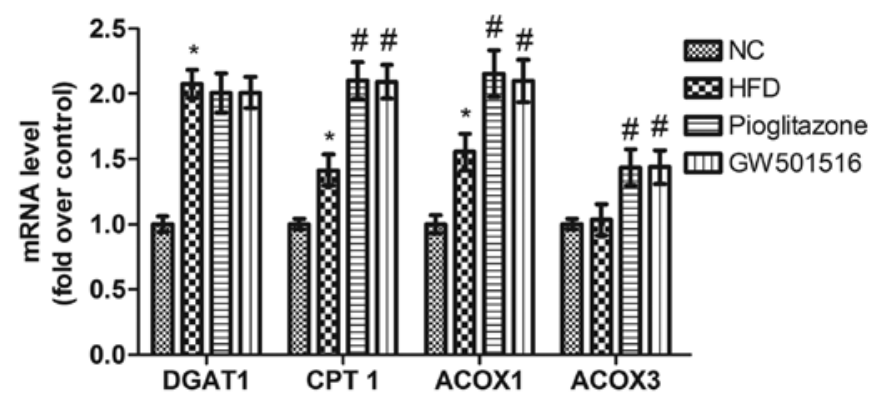

Figure 7. Total RNA was extracted from the livers of the rats undergoing the different treatments. The mRNA levels of diglyceride acyltransferase 1 (DGAT1), carnitin palmitoyltransferase I (CPT-I), acyl-CoA oxidase (ACOX)1 and ACOX3 were determined by RT-qPCR. All data were normalized based on $\beta$-actin levels and are expressed as the means \pm SEM. "P<0.05, compared to the normal control (NC) group; ${ }^{~} \mathrm{P}<0.05$, compared to the high-fat diet (HFD) group.

ment with GW501516, as CPT-1 is the rate-limiting enzyme for $\beta$-oxidation in the mitochondria (38), and ACOX1/ACOX3 are the rate-limiting enzymes for $\beta$-oxidation in peroxisomes (39). 


\section{Discussion}

NAFLD is an increasingly common condition, characterized by the increased accumulation of lipids in the liver, which may lead to the development of progressive hepatic fibrosis, cirrhosis and hepatic failure. The increase in fat deposits in the liver can be caused by an increase in the uptake of free fatty acids into the liver, impaired fatty acid $\beta$-oxidation, or the increased incidence of de novo lipogenesis (40). NAFLD is closely related to obesity, hyperlipidemia and insulin resistance, as well as other other metabolic disorders in the liver $(1,2)$. A recent study emphasized the important role that PPARs play in certain metabolic disorders and NAFLD (41). PPARs, which are known to be important regulators of energy metabolism and homeostasis, play a role in modulating the accumulation of TGs in the liver, which is a hallmark of the development of NAFLD $(32,41,42)$.

GW501516, as a potent agonist of PPAR $\delta$, regulates glycometabolism, fatty acid metabolism and insulin resistance (22-24). GW501516 has been shown to enhance the $\beta$-oxidation of liver fatty acids by increasing the expression of PPAR $\alpha$-target genes involved in fatty acid oxidation (23). Several studies have highlighted that insulin resistance is a main characteristic of NAFLD $(1,7,8)$. In skeletal muscle cells, treatment with GW501516 has been shown to prevent fatty acid-induced inflammation and insulin resistance in by preventing the phosphorylation of insulin receptor substrate-1 at Ser307 and the inhibition of insulin-stimulated Akt phosphorylation, which is caused by exposure to the saturated fatty acid, palmitate (22). The expression of two well-known PPARd target genes involved in fatty acid oxidation, CPT-1 and pyruvate dehydrogenase kinase 4, has also been shown to be elevated following treatment with GW501516 (22). In human liver cells, treatment with GW501516 has been shown to increase the adenosine monophosphate (AMP)/adenosine triphosphate (ATP) ratio and decrease the ATP/adenosine diphosphate (ADP) ratio, thus contributing to the prevention of insulin resistance (24). GW501516 can also prevent endoplasmic reticulum stress-associated inflammation and insulin resistance in skeletal muscle cells by activating AMPK $(43,44)$. The Akt and ERK signaling pathways have also been shown to be involved in the effects of GW501516 (45). In a previous study, in INS-1E cells treated with GW501516, the expression of v-maf avian musculoaponeurotic fibrosarcoma oncogene homolog A (MafA), GLUT-2, and the upregulation of insulin, indicated that the c-Jun N-terminal kinase (JNK)-MafA-GLUT-2 pathway was involved in the enhanced effects of PPAR $\delta$ on insulin secretion (46). GW501516 also possesses the ability to reduce the proliferative potential of hepatoma cells (47). All these studies suggested that GW501516 alleviates NAFLD, as GW501516 is able to increase fatty acid oxidation, reduce insulin resistance, prevent endoplasmic reticulum stress and hepatocyte proliferation and induce insulin secretion. However, GW501516 cannot diminish the progression of liver fibrosis. The induction of hepatic stellate cell (HSC) apoptosis is a potent strategy used to diminish the progression of liver fibrosis. However, GW501516-activated PPAR $\beta / \delta$ promotes liver fibrosis through the p38-JNK MAPK-induced HSC proliferation (48), and decreases the soluble egg antigen (SEA)-induced HSC apoptosis (49). However, KD3010, another agonist of PPARS, has been shown to have hepatoprotective and antifibrotic effects in hepatocytes and in a model of cholestasis-induced liver injury and fibrosis (50). Therefore, the effects of GW501516 on NAFLD are still debatable.

In the present study, we demonstrated that treatment with GW501516 significantly alleviated the pathological changes in the livers of rats in our model of HFD-induced NAFLD. GW501516 significantly decreased the HOMA-IR index, and increased the levels of IGF-1 in the rats with HFD-induced NAFLD, indicating that insulin resistance was reduced by GW501516. This finding was consistent with that of previous studies $(22,24,43)$. We also demonstrated that the protective effects of GW501516 were more potent than those of pioglitazone. The decreased expression of GLUT-2 in the livers of the rats in our NAFLD model was significantly increased following treatment with GW501516, almost to normal levels (Fig. 6B). Cao et al (46) demonstrated that GW501516 upregulated the expression of GLUT-2 through the JNK-MafA-GLUT-2 pathway. These results suggest that glucose metabolism is modulated by GW501516. In our study, the expression of SREBP-1c, a key lipogenic transcription factor involved in lipogenesis, was also reduced to normal levels following treatment with GW501516 (Fig. 6A). We also demonstrated that the mRNA levels of DGAT1 were not significantly altered, but those of CPT-1, ACOX1 and ACOX3 were all significantly upregulated following treatment with GW501516 (Fig. 7). DGAT1 catalyzes the formation of TGs from diacylglycerol and Acyl-CoA (37). This is considered the terminal and only committed step in TG synthesis. CPT-1 is the rate-limiting enzyme involved in the $\beta$-oxidation of long-chain fatty acids (38). ACOX1 catalyzes the first, rate-limiting step in peroxisome $\beta$-oxidation, of medium to very long straight-chain fatty acids, and ACOX3 is involved in the desaturation of branched fatty acids in peroxisomes (39). Our observations indicated that TG synthesis was not significantly affected by GW501516 treatment, but that the $\beta$-oxidation of fatty acids was significantly enhanced. This observation was in line with that of a previous study, in which fatty acid oxidation was increased by the activation of PPAR $\delta$ in human myotubes (51).

Although we demonstrated that treatment with GW501516 alleviated NAFLD by modulating glucose and lipid metabolism, the contribution of the activation of PPAR $\delta$ to this process remains to be clarified, as PPAR $\alpha$ and PPAR $\gamma$ may also be activated by GW501516. However, it has been suggested that PPAR $\delta$ plays a major role in the effects of GW501516 on NAFLD since GW501516 displays a high affinity $(\mathrm{Ki}=1 \mathrm{nM})$ and potency $\left(\mathrm{EC}_{50}=1 \mathrm{nM}\right)$ for PPAR $\delta$ that is 1,000 -fold greater than that for PPAR $\alpha$ and PPAR $\gamma$ (52).

In conclusion, in the present study, we demonstrated that GW501516 prevented the progression of NAFLD in our rat model of HFD-induced NAFLD. GW501516 improved glucose and fatty acid metabolism by modulating the levels of glucose and fatty acid metabolic enzymes. Our results thus suggest that treatment with GW501516 may prove to be a potential therapeutic strategy for NAFLD.

\section{Acknowledgements}

The present study was supported by a grant from the National Natural Science Foundation of China (no. 81070328). 


\section{References}

1. Sanyal AJ, Campbell-Sargent C, Mirshahi F, Rizzo WB, Contos MJ, Sterling RK, Luketic VA, Shiffman ML and Clore JN: Nonalcoholic steatohepatitis: association of insulin resistance and mitochondrial abnormalities. Gastroenterology 120: 1183-1192, 2001.

2. Sanyal AJ; American Gastroenterological Association: AGA technical review on nonalcoholic fatty liver disease. Gastroenterology 123: 1705-1725, 2002.

3. Day CP: Pathogenesis of steatohepatitis. Best Pract Res Clin Gastroenterol 16: 663-678, 2002.

4. Browning JD, Szczepaniak LS, Dobbins R, Nuremberg P, Horton JD, Cohen JC, Grundy SM and Hobbs HH: Prevalence of hepatic steatosis in an urban population in the United States: impact of ethnicity. Hepatology 40: 1387-1395, 2004.

5. Gaggini M, Morelli M, Buzzigoli E, DeFronzo RA, Bugianesi E and Gastaldelli A: Non-alcoholic fatty liver disease (NAFLD) and its connection with insulin resistance, dyslipidemia, atherosclerosis and coronary heart disease. Nutrients 5: 1544-1560, 2013.

6. Williams CD, Stengel J, Asike MI, Torres DM, Shaw J, Contreras M, Landt CL and Harrison SA: Prevalence of nonalcoholic fatty liver disease and nonalcoholic steatohepatitis among a largely middle-aged population utilizing ultrasound and liver biopsy: a prospective study. Gastroenterology 140: 124-131, 2011.

7. Yki-Jarvinen $\mathrm{H}$ : Liver fat in the pathogenesis of insulin resistance and type 2 diabetes. Dig Dis 28: 203-209, 2010.

8. Fabbrini E, Magkos F, Mohammed BS, Pietka T, Abumrad NA, Patterson BW, Okunade A and Klein S: Intrahepatic fat, not visceral fat, is linked with metabolic complications of obesity. Proc Natl Acad Sci USA 106: 15430-15435, 2009.

9. Yki-Jarvinen H: Fat in the liver and insulin resistance. Ann Med 37: 347-356, 2005

10. Desvergne B and Wahli W: Peroxisome proliferator-activated receptors: nuclear control of metabolism. Endocr Rev 20: 649-688, 1999.

11. Aoyama T, Peters JM, Iritani N, Nakajima T, Furihata K, Hashimoto T and Gonzalez FJ: Altered constitutive expression of fatty acid-metabolizing enzymes in mice lacking the peroxisome proliferator-activated receptor alpha (PPARalpha). J Biol Chem 273: 5678-5684, 1998.

12. Fan CY, Pan J, Chu R, Lee D, Kluckman KD, Usuda N, Singh I, Yeldandi AV, Rao MS, Maeda N and Reddy JK: Hepatocellular and hepatic peroxisomal alterations in mice with a disrupted peroxisomal fatty acyl-coenzyme A oxidase gene. J Biol Chem 271: 24698-24710, 1996.

13. Leone TC, Weinheimer CJ and Kelly DP: A critical role for the peroxisome proliferator-activated receptor alpha (PPARalpha) in the cellular fasting response: the PPARalpha-null mouse as a model of fatty acid oxidation disorders. Proc Natl Acad Sci USA 96: 7473-7478, 1999.

14. Tontonoz P, Hu E and Spiegelman BM: Stimulation of adipogenesis in fibroblasts by PPAR gamma 2, a lipid-activated transcription factor. Cell 79: 1147-1156, 1994.

15. Larsen TM, Toubro S and Astrup A: PPARgamma agonists in the treatment of type II diabetes: is increased fatness commensurate with long-term efficacy? Int J Obes Relat Metab Disord 27: 147-161, 2003 .

16. Seo YS, Kim JH, Jo NY, Choi KM, Baik SH, Park JJ, Kim JS, Byun KS, Bak YT, Lee CH, et al: PPAR agonists treatment is effective in a nonalcoholic fatty liver disease animal model by modulating fatty-acid metabolic enzymes. J Gastroenterol Hepatol 23: 102-109, 2008.

17. Gillies PS and Dunn CJ: Pioglitazone. Drugs 60: 333-345, 2000.

18. Smith U: Pioglitazone: mechanism of action. Int J Clin Pract Suppl: 13-18, 2001.

19. Vamecq $\mathbf{J}$ and Latruffe N: Medical significance of peroxisome proliferator-activated receptors. Lancet 354: 141-148, 1999.

20. Neuschwander-Tetri BA, Brunt EM, Wehmeier KR, Oliver D and Bacon BR: Improved nonalcoholic steatohepatitis after 48 weeks of treatment with the PPAR-gamma ligand rosiglitazone. Hepatology 38: 1008-1017, 2003.

21. Oger F, Dubois-Chevalier J, Gheeraert C, Avner S, Durand E, Froguel P, Salbert G, Staels B, Lefebvre P and Eeckhoute J: Peroxisome proliferator-activated receptor gamma regulates genes involved in insulin/insulin-like growth factor signaling and lipid metabolism during adipogenesis through functionally distinct enhancer classes. J Biol Chem 289: 708-722, 2014.
22. Coll T, Alvarez-Guardia D, Barroso E, Gómez-Foix AM, Palomer X, Laguna JC and Vázquez-Carrera M: Activation of peroxisome proliferator-activated receptor- $\{$ delta\} by GW501516 prevents fatty acid-induced nuclear factor- $\{$ kappa $\}$ B activation and insulin resistance in skeletal muscle cells. Endocrinology 151: 1560-1569, 2010

23. Barroso E, Rodriguez-Calvo R, Serrano-Marco L, Astudillo AM, Balsinde J, Palomer X and Vázquez-Carrera M: The PPARbeta/delta activator GW501516 prevents the down-regulation of AMPK caused by a high-fat diet in liver and amplifies the PGC-1alpha-Lipin 1-PPARalpha pathway leading to increased fatty acid oxidation. Endocrinology 152: 1848-1859, 2011.

24. Serrano-Marco L, Barroso E, El Kochairi I, Palomer X, Michalik L, Wahli W and Vázquez-Carrera M: The peroxisome proliferator-activated receptor (PPAR) beta/delta agonist GW501516 inhibits IL-6-induced signal transducer and activator of transcription 3 (STAT3) activation and insulin resistance in human liver cells. Diabetologia 55: 743-751, 2012.

25. Nagasawa T, Inada Y, Nakano S, Tamura T, Takahashi T, Maruyama K, Yamazaki Y, Kuroda J and Shibata N: Effects of bezafibrate, PPAR pan-agonist, and GW501516, PPARdelta agonist, on development of steatohepatitis in mice fed a methionine- and choline-deficient diet. Eur J Pharmacol 536: 182-191, 2006.

26. Matthews DR, Hosker JP, Rudenski AS, Naylor BA, Treacher DF and Turner RC: Homeostasis model assessment: insulin resistance and beta-cell function from fasting plasma glucose and insulin concentrations in man. Diabetologia 28: 412-419, 1985.

27. Wang HN, Wang YR, Liu GQ, Liu Z, Wu PX, Wei XL and Hong TP: Inhibition of hepatic interleukin-18 production by rosiglitazone in a rat model of nonalcoholic fatty liver disease. World J Gastroenterol 14: 7240-7246, 2008.

28. Livak KJ and Schmittgen TD: Analysis of relative gene expression data using real-time quantitative PCR and the 2(-Delta Delta C(T)) Method. Methods 25: 402-408, 2001.

29. Ferre P and Foufelle F: Hepatic steatosis: a role for de novo lipogenesis and the transcription factor SREBP-1c. Diabetes Obes Metab 12 (Suppl 2): S83-S92, 2010.

30. Shimano H, Yahagi N, Amemiya-Kudo M, Hasty AH, Osuga J, Tamura Y, Shionoiri F, Iizuka Y, Ohashi K, Harada K, et al: Sterol regulatory element-binding protein-1 as a key transcription factor for nutritional induction of lipogenic enzyme genes. J Biol Chem 274: 35832-35839, 1999.

31. Keller H, Dreyer C, Medin J, Mahfoudi A, Ozato K and Wahli W: Fatty acids and retinoids control lipid metabolism through activation of peroxisome proliferator-activated receptor-retinoid $\mathrm{X}$ receptor heterodimers. Proc Natl Acad Sci USA 90: 2160-2164, 1993.

32. Keller H, Mahfoudi A, Dreyer C, Hihi AK, Medin J, Ozato K and Wahli W: Peroxisome proliferator-activated receptors and lipid metabolism. Ann NY Acad Sci 684: 157-173, 1993.

33. Keller $\mathrm{H}$ and Wahli W: Peroxisome proliferator-activated receptors A link between endocrinology and nutrition? Trends Endocrinol Metab 4: 291-296, 1993.

34. Kliewer SA, Sundseth SS, Jones SA,Brown PJ, Wisely GB, Koble CS, Devchand P, Wahli W, Willson TM, Lenhard JM and Lehmann JM: Fatty acids and eicosanoids regulate gene expression through direct interactions with peroxisome proliferator-activated receptors alpha and gamma. Proc Natl Acad Sci USA 94: 4318-4323, 1997.

35. Forman BM, Chen J and Evans RM: Hypolipidemic drugs, polyunsaturated fatty acids, and eicosanoids are ligands for peroxisome proliferator-activated receptors alpha and delta. Proc Natl Acad Sci USA 94: 4312-4317, 1997.

36. Lee CH, Olson P, Hevener A, Mehl I, Chong LW, Olefsky JM, Gonzalez FJ, Ham J, Kang H, Peters JM and Evans RM: PPARdelta regulates glucose metabolism and insulin sensitivity. Proc Natl Acad Sci USA 103: 3444-3449, 2006.

37. Cases S, Smith SJ, Zheng YW, Myers HM, Lear SR, Sande E, Novak S, Collins C, Welch CB, Lusis AJ, et al: Identification of a gene encoding an acyl CoA: diacylglycerol acyltransferase, a key enzyme in triacylglycerol synthesis. Proc Natl Acad Sci USA 95: 13018-13023, 1998.

38. Eaton S: Control of mitochondrial beta-oxidation flux. Prog Lipid Res 41: 197-239, 2002.

39. Poirier Y, Antonenkov VD, Glumoff T and Hiltunen JK: Peroxisomal beta-oxidation - a metabolic pathway with multiple functions. Biochim Biophys Acta 1763: 1413-1426, 2006. 
40. Koo SH: Nonalcoholic fatty liver disease: molecular mechanisms for the hepatic steatosis. Clin Mol Hepatol 19: 210-215, 2013.

41. Tailleux A, Wouters K and Staels B: Roles of PPARs in NAFLD: potential therapeutic targets. Biochim Biophys Acta 1821: 809-818, 2012

42. Wahli W and Michalik L: PPARs at the crossroads of lipid signaling and inflammation. Trends Endocrinol Metab 23: 351-363, 2012.

43. Salvado L, Barroso E, Gomez-Foix AM, Palomer X, Michalik L, Wahli W and Vázquez-Carrera M: PPARbeta/delta prevents endoplasmic reticulum stress-associated inflammation and insulin resistance in skeletal muscle cells through an AMPK-dependent mechanism. Diabetologia 57: 2126-2135, 2014.

44. Palomer X, Capdevila-Busquets E, Botteri G, Salvadó L, Barroso E, Davidson MM, Michalik L, Wahli W and VázquezCarrera M: PPARbeta/delta attenuates palmitate-induced endoplasmic reticulum stress and induces autophagic markers in human cardiac cells. Int J Cardiol 174: 110-118, 2014.

45. Ding H, Zhang Y, Liu L, Yuan H, Qu J and Shen R: Activation of peroxisome proliferator activator receptor delta in mouse impacts lipid composition and placental development at early stage of gestation. Biol Reprod 91: 57, 2014.

46. Cao M, Long Y, Tong Y, Wan J and Tong N: Activation of PPARdelta up-regulates the expression of insulin gene transcription factor MafA and ameliorates glucose-induced insulin secretion impaired by palmitate. Mol Cell Biochem 366: 183-189, 2012.

47. Vacca M, D'Amore S, Graziano G, D’Orazio A, Cariello M, Massafra V, Salvatore L, Martelli N, Murzilli S, Lo Sasso G, et al: Clustering nuclear receptors in liver regeneration identifies candidate modulators of hepatocyte proliferation and hepatocarcinoma. PLoS One 9: e104449, 2014.
48. Kostadinova R, Montagner A, Gouranton E, Fleury S, Guillou H, Dombrowicz D, Desreumaux P and Wahli W: GW501516-activated PPARbeta/delta promotes liver fibrosis via p38-JNK MAPK-induced hepatic stellate cell proliferation. Cell Biosci 2: 34, 2012.

49. Wang J, Xu F, Zhu D, Duan Y, Chen J, Sun X, He X, Li P, Sun W and Feng J: Schistosoma japonicum soluble egg antigens facilitate hepatic stellate cell apoptosis by downregulating Akt expression and upregulating p53 and DR5 expression. PLoS Negl Trop Dis 8: e3106, 2014

50. Iwaisako K, Haimerl M, Paik YH, Taura K, Kodama Y, Sirlin C, Yu E, Yu RT, Downes M, Evans RM, et al: Protection from liver fibrosis by a peroxisome proliferator-activated receptor delta agonist. Proc Natl Acad Sci USA 109: E1369-E1376, 2012.

51. Feng YZ, Nikolic N, Bakke SS, Boekschoten MV, Kersten S, Kase ET, Rustan AC and Thoresen GH: PPARdelta activation in human myotubes increases mitochondrial fatty acid oxidative capacity and reduces glucose utilization by a switch in substrate preference. Arch Physiol Biochem 120: 12-21, 2014.

52. Oliver WR Jr, Shenk JL, Snaith MR, Russell CS, Plunket KD, Bodkin NL, Lewis MC, Winegar DA, Sznaidman ML, Lambert MH, et al: A selective peroxisome proliferator-activated receptor delta agonist promotes reverse cholesterol transport. Proc Natl Acad Sci USA 98: 5306-5311, 2001. 